von $\mathrm{NaCl}$ und $\mathrm{KCl}$ gegenüber $\mathrm{Tl}$ und $\mathrm{Mn}$ wird also gittertheoretisch und energetisch gefordert.

Was die Absolutgröße der Energietönungen (7) und (11) anbelangt, so ist ihre Größenordnung richtig. Die Genauigkeit des Verfahrens dürfte einige Zehntel $\mathrm{eV}$ betragen und damit in der Größenordnung der Beträge selbst liegen. Gegenüber den bisherigen Verfahren, bei denen die Gitterverzerrung infolge abweichender Größe des Störions nicht oder ungenügend berücksichtigt wurde, ist die Genauigkeit aber doch sehr erheblich gesteigert, denn diese lieferten auch die Größenordnung meist unrichtig. Die absolute Genauigkeit reicht ohne weiteres aus, um z. B. auszuschließen, daß das $\mathrm{Mn}^{++}$ im $\mathrm{KCl}$ zwar eingebaut, aber $\mathrm{zu} \mathrm{Mn}^{+}$umgeladen wird - ähnlich der Umladung des $\mathbf{E u}^{+++} \mathrm{zu} \mathrm{Eu}^{++}$ in verschiedenen Erdalkalichalkogeniden ${ }^{10}$ - und deshalb nicht leuchtet. Die Rechnung ergibt dafür einen um einige $\mathrm{eV}$ höheren Energieaufwand.

Eine weitere Steigerung der Genauigkeit des gittertheoretischen Teils der Rechnung scheint uns vorläufig nicht gerechtfertigt, da die übrigen Energiebeträge nicht sicher genug bekannt sind. Z. B.

10 P. Brauer, Z. Naturforschg: 6a, 561, 562 [1951].

11 H.W. Etzel u. R. J. Maurer, J. chem. Physics 18, 1003 [1950]. gilt die in Tab. 2 (11) angegebene Energietönung in wässeriger Lösung eigentlich für verdünnte Lösungen; für die an $\mathrm{MeCl}$ gesättigten Lösungen wäre die Solvatationsenergie von $\mathrm{MeCl}$ gleich der Gitterenergie oder in Tab. 2 wäre (9) gleich null zu setzen. (11) stellt somit einen Grenzwert dar. Die wirkliche Energietönung in wässeriger Lösung dürfte zwischen (7) und (11) liegen. Nimmt man für das in sehr geringer Konzentration anwesende $\mathrm{MnCl}_{2}$ die Verhältnisse in verdünnter Lösung als gültig an, so würde in Tab. 2 (11) sich ergeben $-0,46 \mathrm{eV}$ (statt 0,38) bzw. $+0,48 \mathrm{eV}$ (statt 0,86 ), was an unserer Aussage nichts Wesentliches ändert.

In der Aufstellung Tab. 2 haben wir nicht berücksichtigt, daß sich vermutlich je ein eingebautes $\mathrm{Mn}^{++}$und die restliche $\mathrm{K}^{+}$-Lücke unter dem Einfluß Coulombscher Anziehung assoziieren würden. Die hierbei zu gewinnende Energie dürfte etwa 0,3 bis $0,5 \mathrm{eV}$ betragen, wenn man die an $\mathrm{Cd}^{++}$in $\mathrm{NaCl}$ experimentell von Etzel und Maurer ${ }^{11}$ und theoretisch von Reitz und Gammel ${ }^{12}$ gefundenen Werte überträgt. Das würde aber an unseren Aussagen auch nichts Wesentliches ändern.

12 J.R. Reitz u. J.L. Gammel, J. chem. Physics 19, 894 [1951].

\title{
Über neue halbleitende Verbindungen
}

\begin{abstract}
Von H. WELKER
Aus dem Allgemeinen Laboratorium der Siemens-Schuckertwerke in Erlangen

(Z. Naturforschg. 7 a, 744-749 [1952]; eingegangen am 25. August 1952)

Die vorliegende Arbeit beschäftigt sich mit der Nachbildung der halbleitenden Elemente der vierten Hauptgruppe ${ }^{1}$ des periodischen Systems (Diamant, Si, Ge, graues Sn) durch bisher nicht als Halbleiter bekannte Verbindungen von Elementen der dritten Hauptgruppe ( $\mathrm{Al}, \mathrm{Ga}, \mathrm{In})$ mit Elementen der fünften Hauptgruppe ( $\mathrm{P}, \mathrm{As}, \mathrm{Sb})$. Infolge der quantenmechanischen Resonanz zwischen homöopolarem und heteropolarem Bindungsanteil lassen sich Voraussagen über die Halbleitereigenschaften dieser Verbindungen machen, die mit den Experimenten in gutem Einklang sind. Es zeigt sich dabei, daß diese Verbindungen in einigen Eigenschaften noch weit über die Elemente der vierten Gruppe hinausgehen. Z. B. werden bei In Sb Elektronenbeweglichkeiten bis zu $25000 \mathrm{~cm}^{2} /$ Volt sec gemessen.
\end{abstract}

$\mathrm{I}_{\mathrm{r}}^{\mathrm{n}}$ $\mathrm{n}$ den letzten Jahren hat sich die physikalische Forschung auf dem Halbleitergebiet sehr intensiv mit den Elementen der vierten Gruppe des periodischen Systems (Diamant, Si, Ge, graues Sn) be-

1 Hier werden, wie dies neuerdings üblich ist, die Elemente B, Al, Ga, In, Tl als zur dritten Hauptgruppe, die Elemente C, Si, Ge, Sn, $\mathrm{Pb}$ als zur vierten Hauptgruppe, die Elemente N, P, As, Sb, Bi als zur fünften Hauptgruppe gehörig bezeichnet. In der älteren Lite- schäftigt. Vor allem haben Silicium und Germanium wegen ihrer Gleichrichtereigenschaften eine besondere Bedeutung erlangt; letzteres insbesondere, weil es der Ausgangspunkt für die Entdeckung des Tran-

ratur wird davon abweichend verfahren, z. B. bei Van Arkel, Reine Metalle, Springer, Berlin 1939. Im weiteren Verlauf der Arbeit werden wir von Gruppe schlechthin sprechen, da Verwechslungen nicht möglich sind. 
sistoreffektes war. Die vier genannten Stoffe besitzen als gemeinsames Kennzeichen das Diamantgitter mit der charakteristischen Eigenschaft, daß die vier nächsten Nachbarn eines herausgegriffenen Atoms auf den Eckpunkten eines gleichseitigen Tetraeders liegen. Die Atome werden durch homöopolare Bindungen zusammengehalten, die brückenartig zwischen nächsten Nachbarn wirksam sind. Jede solche Brücke ist durch zwei Elektronen besetzt, die nicht zur elektrischen Leitfähigkeit beitragen. In engem Zusammenhang mit diesen homöopolaren Bindungsverhältnissen steht die hohe Beweglichkeit lichtelektrisch ausgelöster oder durch Störstellen gebildeter Elektronen in diesen Körpern. Die Beweglichkeiten betragen bei Zimmertemperatur $900 \mathrm{~cm}^{2} /$ Volt sec für Diamant ${ }^{2}, 1200$ für Silicium $^{3}, 3600$ für Germanium ${ }^{4}$ und ca. 3000 für graues Zinn ${ }^{5}$. Diese hohen Beweglichkeiten hängen mit zwei typischen Eigenschaften des Diamantgitters zusammen. Infolge der hohen Bindungsfestigkeit im Diamantgitter sind die Amplituden der thermischen Gitterschwingungen besonders klein. In zweiter Linie dürfte die Existenz der homöopolaren Brücken zu einer kleinen scheinbaren Masse $m^{*}$ der Leitungselektronen führen und damit auch von dieser Seite her eine hohe Beweglichkeit begünstigen.

Eine weitere, für die halbleitenden Eigenschaften sehr wichtige Größe ist die Breite der für Elektronen verbotenen Zone. Sie nimmt mit wachsender Ordnungszahl dauernd ab und beträgt $6-7 \mathrm{eV}$ beim Diamant, 1,1 eV bei Silicium, 0,7 eV bei Germanium und $0,1 \mathrm{eV}$ beim grauen Zinn. Die Breite der verbotenen Zone besitzt denselben Gang mit der Ordnungszahl wie die Schmelzpunkte dieser Stoffe $(\mathrm{C} \approx$ $3800^{\circ} \mathrm{C}, \quad \mathrm{Si}=1420^{\circ} \mathrm{C}, \quad \mathrm{Ge}=958^{\circ} \mathrm{C}$ und $\mathrm{Sn}=$ $\left.232^{\circ} \mathrm{C}\right)$.

Dieser Zusammenhang ist innerhalb einer homologen Reihe theoretisch verständlich. Einerseits liegt der Schmelzpunkt um so höher, je größer die Bindungsfestigkeit des betreffenden Elementes ist. Andererseits ist nach der Elektronentheorie der Metalle die Breite der verbotenen Zone um so größer, je größer im periodischen Gitterpotential der Potentialunterschied zwischen dem Maximum

${ }^{2}$ Vgl. C. C. Klick u. R. J. Maurer, Physic. Rev. 81, 124 [1951].

${ }^{3}$ Vgl. G. K. Teal u. E. Buehler, Physic. Rev. 87, 190 [1952]. J. R. Haynes u. W. C. Westphal, Physic. Rev. 85, 680 [1952].

4 J. R. Haynes u. W. Shockley, Physic. Rev. 81, 835 [1951]. und dem Minimum des Potentials ist. Da diese Potentialdifferenz bei großer Bindungsfestigkeit größer ist als bei kleiner Bindungsfestigkeit, ergibt sich, daß die Breite der verbotenen Zone mit der Bindungsfestigkeit wächst.

Eine weitere bemerkenswerte Eigenschaft der halbleitenden Elemente der vierten Gruppe besteht auch darin, daß die Abhängigkeit der Leitungseigenschaften von eingebauten Störstellen besonders übersichtlich ist. So sind die Elemente der dritten Gruppe (B, Al, Ga, In) als Acceptoren, die der fünften Gruppe (N, P, As, Sb) als Donatoren wirksam. Innerhalb der homologen Reihen der Acceptoren und Donatoren besteht noch eine weitere Differenzierung, die durch die covalenten Radien der Störatome gegeben ist. So wird Ga (covalenter Radius 1,26$)$ leichter eingebaut als In $(1,44)$, As $(1,18)$ leichter als $\mathrm{Sb}(1,36)$.

Es erhebt sich nun die Frage, ob es andere Körper gibt, welche die oben genannten günstigen Eigenschaften in gleichem Maße oder sogar noch ausgeprägter besitzen. Wenn wir uns auf der Suche nach solchen Körpern von dem Prinzip leiten lassen, von den halbleitenden Elementen der vierten Gruppe nur so wenig als möglich abzuweichen, so werden wir dazu geführt, die Verbindungen der Elemente der dritten und fünften Gruppe des periodischen Systems, kurzVerbindungen $\mathrm{A}^{\mathrm{III}} \mathrm{B}^{\mathrm{V}}$ genannt, zu untersuchen.

Von den Elementen der dritten Gruppe kommen dabei in erster Linie in Frage die Elemente Al, Ga und In,von denen der fünften Gruppe die Elemente $\mathrm{P}$, As und Sb. Die 9 Verbindungen ${ }^{6} \mathrm{AlP}, \mathrm{AlAs}$, AlSb, GaP, GaAs, GaSb, InP, InAs, InSb besitzen die Zinkblendestruktur mit ihrer großen Verwandtschaft zur Diamantstruktur. Läßt man nämlich in der Zinkblendestruktur gedanklich die beiden Verbindungspartner miteinander identisch werden, so kommt die Diamantstruktur heraus. Würden wir noch leichtere Elemente $(\mathrm{B}, \mathrm{N})$ in die Betrachtung einbeziehen, so würde sich in den entsprechenden Kombinationen bereits das Wurzitgitter durchsetzen. Dieses ist dem Zinkblendegitter noch sehr verwandt, und die betreffenden Verbindungen haben auch Halbleitercharakter. Die Verbindungen be-

${ }^{5}$ Vgl. G. Busch, I. Wieland u. H. Zoller, Helv. physica Acta 24, 49 [1951].

6 Diese Verbindungen fallen, soweit ihre Komponenten als Metalle anzusehen sind, unter den Sammelbegriff ,,intermetallische Verbindungen". 
sitzen aber nicht mehr die übersichtliche Eigenschaft der kubischen Isotropie, sondern sind anisotrop hexagonal. Würden wir noch schwerere Elemente ins Auge fassen ( $\mathrm{Tl}, \mathrm{Bi}$ ), so würden sich in den Kombinationen der metallische Charakter durchsetzen und damit die halbleitenden Eigenschaften verloren gehen.

Die Nachbildung der vierwertigen Elemente durch Verbindungen $\mathrm{A}^{\mathrm{III}} \mathrm{B}^{\mathrm{v}}$ ist nicht nur hinsichtlich der Struktur eine besonders enge, sondern auch hinsichtlich des Gitterabstandes. So ist der Abstand nächster Nachbarn im Si 2,34 $\AA$, während seine Nachbildung, das AlP, den Abstand 2,36 § besitzt. Ähnliches gilt sowohl für Germanium $(2,44 \AA)$ und GaAs $(2,44 \AA)$ als auch für graues Sn $(2,80)$ und InSb $(2,80 \AA)$. Diese einfachen Zusammenhänge haben es Pauling ${ }^{7}$ ermöglicht, ein System covalenter Radien aufzustellen, mit welchen sich die Gitterabstände der Elemente der vierten Gruppe und der oben genannten 9 Verbindungen darstellen lassen. (Vgl. dazu Abb. 1, linke Reihe, in welcher die covalenten Tetraederradien jeder Verbindung maßstabgetreu eingetragen sind.) Der kürzeste Atomabstand ist die Summe der covalenten Radien. Rechnung und Messung stimmen dabei aufs beste überein.

In einem weiteren Sinne können die Körper AlSb mit 2,62 $\AA$ und InP mit 2,54 $\AA$ dem Ge zugeordnet werden, während die Körper AlAs mit 2,44 $\AA$ und GaP mit 2,36 $\AA$ einer hypothetischen. Verbindung SiGe mit 2,39 $\AA$, die Körper GaSb mit 2,62 $\AA$ und InAs mit 2,62 $\AA$ einer hypothetischen Verbindung GeSn mit 2,62 $\AA$ zugeordnet werden können.

Wie die gute Darstellbarkeit der Abstandsverhältnisse durch das System covalenter Radien beweist, kann in den Verbindungen $\mathrm{A}^{\mathrm{III}} \mathrm{B}^{\mathrm{V}}$ die Bindung in erster Näherung als homöopolar angesehen werden, ähnlich wie in den Elementen. Da jedoch die Elemente der fünften Gruppe stärker elektronegativ und die der dritten Gruppe schwächer elektronegativ sind als die entsprechenden Elemente der vierten Gruppe, ist der heteropolare oder Ionen-Charakter einer Verbindung $\mathrm{A}^{\mathrm{III}} \mathrm{B}^{\mathrm{V}}$ stärker als der des ent-

${ }^{7}$ L. Pauling, The Nature of the Chemical Bond, Oxford University Press, London 1950.

Es sei in diesem Zusammenhang auf die folgenden grundlegenden Arbeiten hingewiesen: H. G. Grimm u. A. Sommerfeld, Z. Physik 36, 36 [1926]; V. M. Goldschmidt, Chem. Ber. 60, 1288 [1927], und den zusammenfassenden Artikel von H. G. Grimm u. H. Wolff, Atombau und Chemie, Geiger-Scheel, Hdb. Physik, XXIV/2, J. Springer, Berlin 1933.

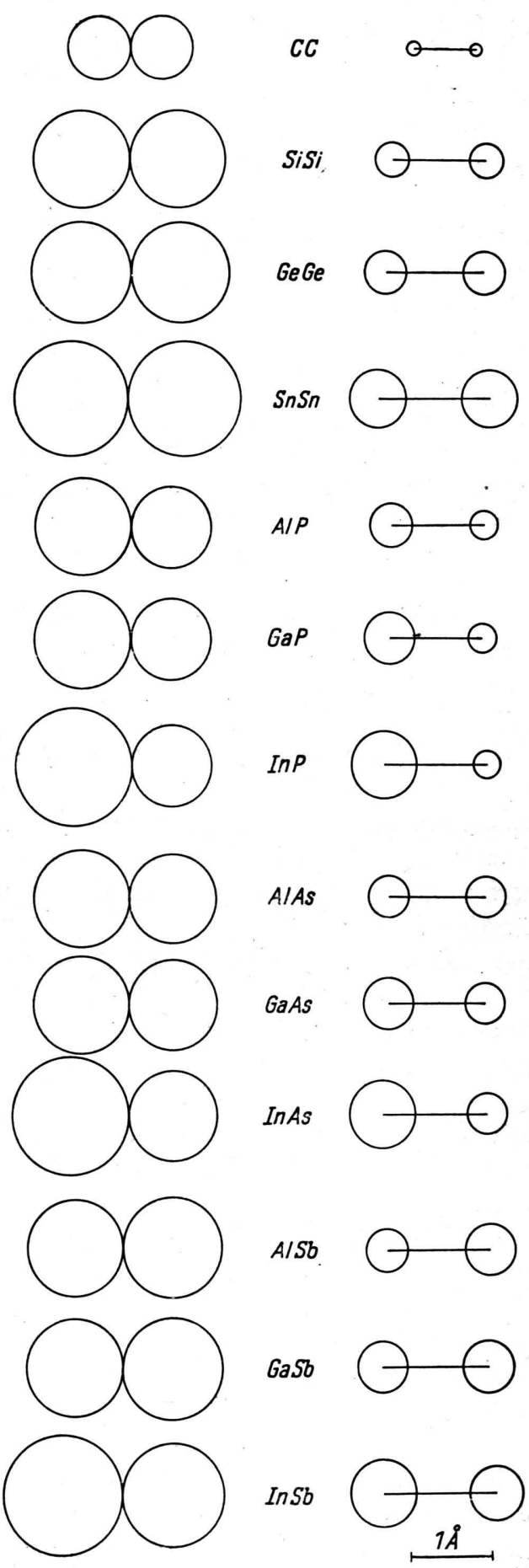

Abb. 1. Maßstabsgetreue Darstellung der Abstandsverhältnisse und Ionengrößen in den Gittern von Diamant, Silicium, Germanium, grauem Zinn und den Verbindungen AIII BV. Linke Reihe: Darstellung mit covalenten Tetraederradien nach Pauling. Rechte Reihe: Darstellung durch Ionen mit Edelgaskonfiguration. 
sprechenden Elementes. Wegen des quantenmechanischen Resonanzprinzipes, welches zwischen dem homöopolaren und dem heteropolaren Anteil der chemischen Bindung wirksam ist, wird bei den Verbindungen $\mathrm{A}^{\mathrm{III}} \mathrm{B}^{\mathrm{V}}$ die Bindungsenergie um einen zusätzlichen Ionenanteil gegenüber den Elementen vergrößert. Dieser zusätzliche Ionenanteil stellt jedoch nur ein Korrekturglied zweiter Ordnung dar. Eine Veranschaulichung der Dichteverhältnisse der Elektronen hat immer noch davon auszugehen, daß die Elemente der dritten Gruppe als dreifach positiv geladene, edelgasähnliche Ionen, die der fünften Gruppe als fünffach positiv geladene, edelgasähnliche Ionen vorliegen und, daß die $3+5=8$ verfügbar werdenden Elektronen die vier homöopolaren Brücken mit den Nachbaratomen bilden. Hingegen würde die extreme Ionenvorstellung davon auszugehen haben, daß die Elemente der dritten Gruppe als dreifach positiv geladene Ionen vorliegen und die der füntten Gruppe als dreifach negativ geladene Ionen. Dieser Bindungsanteil ist aber, wie bereits gesagt, nur zu einem geringen Prozentsatz vertreten.

Abb. 1, rechte Reihe, bringt eine systematische Zusammenstellung der Abstandsverhältnisse der in Frage kommenden Elemente der vierten Gruppe und Verbindungen $\mathrm{A}^{\mathrm{III}} \mathrm{B}^{\mathrm{V}}$, wobei die dritte, vierte und fünfte Gruppe als dreifach, vierfach und fünffach geladene, edelgasähnliche Ionen behandelt sind. Diese Darstellung ist von Interesse, weil sie gestattet, den Gang des Verhältnisses heteropolarer/ homöopolarer Anteil bei den verschiedenen Verbindungen $\mathrm{A}^{\mathrm{III}} \mathrm{B}^{\mathrm{V}} \mathrm{zu}$ erkennen. Z. B. ist bei der Verbindung AlSb das $\mathrm{Al}^{3+}$.Ion (Radius 0,5 $\AA$ ) kleiner als das $\mathrm{Sb}^{5}+$-Ion $(0,62 \AA)$. Die im Vergleich zum $\mathrm{Al}^{3+}$ stärkere Anziehungskraft des $\mathrm{Sb}^{5}+$ auf die Bindungselektronen, welche eine Begünstigung des Ionenanteils in der Bindung bewirkt, erfährt daher eine kompensierendeWirkung durch einen Volumeneffekt. Dieser Volumeneffekt besteht darin, daß die Elektronen wegen des Pauli-Prinzips (vgl. auch das Thomas-Fermische Atommodell) die Tendenz haben, in leere Räume zu gehen. Dadurch wird die anziehende Wirkung des $\mathrm{Sb}^{5}$ t weitgehend kompensiert. Wir können also damit rechnen, daß die Bindung im AlSb weitgehendst homöopolar und der Ionenanteil sehr gering ist. Der Ionenanteil in der Bindung wird dann successive größer, wenn wir zum GaSb, zum InSb oder gar zum InAs übergehen. In letzterem Fall sieht man deutlich, wie die Anziehung des $\mathrm{Sb}^{5}+$-Ions und der Volumeneffekt zusammenwirken.
Die quantenmechanische Resonanzverfestigung bringt bemerkenswerte Konsequenzen für das elektrische Verhalten der Verbindungen $\mathrm{A}^{\mathrm{III}} \mathrm{B}^{\mathrm{V}}$ mit sich. Wir erläutern dies am Beispiel der Verbindung InSb. Da InSb denselben Gitterabstand hat wie graues Sn, da ferner das gemittelte Atomgewicht von In und Sb dem Atomgewicht des Sn gleich ist, ist auch die Dichte des InSb gleich der des grauen Sn. Da jedoch die Bindungsfestigkeit bei der Verbindung größer ist als beim Element, sind die Amplituden der thermischen Gitterschwingungen bei der Verbindung kleiner als beim entsprechenden Element. Daraus ergibt sich, daß die Elektronenbeweglichkeit im InSb größer ist als im grauen Sn. Messungen des elektrischen Widerstandes, des HallEffektes und der Widerstandsänderung im Magnetfeld haben nun für InSb bei Zimmertemperatur tatsächlich Elektronenbeweglichkeiten bis zu 25000 $\mathrm{cm}^{2} /$ Volt sec ergeben, während graues $\mathrm{Sn}$ nur Beweglichkeiten von etwa $3000 \mathrm{~cm}^{2} /$ Volt sec aufweist.

In Abb. 2 sind die Äquipotentiallinien beim Stromdurchgang durch ein stabförmiges InSb-Präparat mit quadratischem Querschnitt, ohne und mit einem Magnetfeld von 10000 Gauß (letzteres senk-

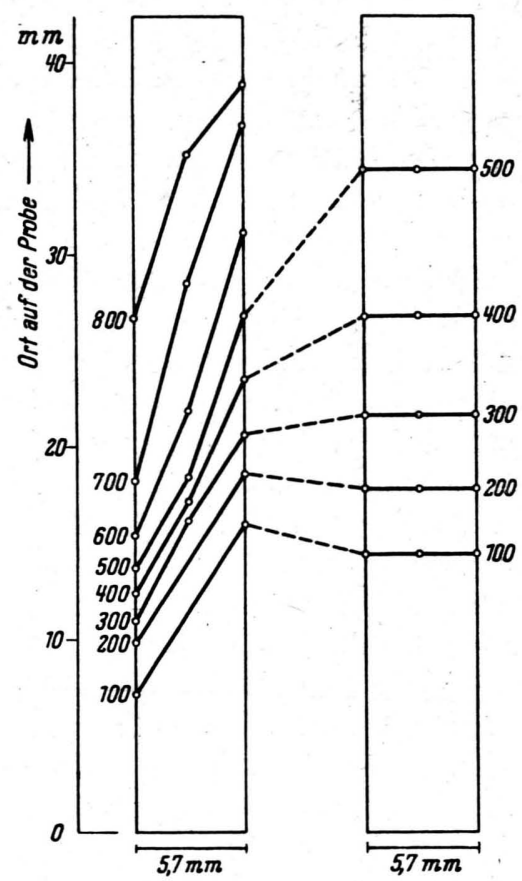

Abb. 2. Verlauf der Äquipotentiallinien in einem stromdurchflossenen InSb-Stab ohne (rechts) und mit (links) Magnetfeld. Die Linien gleichen Potentials in beiden Stäben, sind durch gestrichelte Linien verbunden. Die Zahlen bei den Äquipotentiallinien geben den Wert des Potentials in $\mu$ Volt an. 
recht zur Zeichenebene) dargestellt. Man sieht in der Abbildung deutlich die extrem starke Drehung der Äquipotentiallinien um den Hall-Winkel $\vartheta$, welche durch den Hall-Effekt hervorgerufen wird. Die Elektronenbeweglichkeit kann aus dem Hall-Winkel mittels der bekannten Formel

$$
\vartheta=-\frac{8 \pi}{3} \mu H
$$

( $\mu$ Elektronenbeweglichkeit, $H$ magnetische Feldstärke) berechnet werden. Infolge der Widerstandserhöhung im Magnetfeld werden die Äquipotentiallinien gleichzeitig dichter.

Dieselben Überlegungen sind zutreffend für GaAs im Vergleich zu Ge, und für AlP im Vergleich zu Si. Für die anderen Kombinationen $\mathrm{A}^{\mathrm{III}} \mathrm{B}^{\mathrm{V}}$ fehlt die Vergleichsmöglichkeit mit den Elementen. Grundsätzlich wird man aber ganz allgemein den Satz aufstellen können, daß die Verbindungen $\mathrm{A}^{\mathrm{III}} \mathrm{B}^{\mathrm{V}}$ höhere Elektronenbeweglichkeiten besitzen als die Elemente der vierten Gruppe.

In ähnlicher Weise lassen sich auch Folgerungen ziehen für die Höhe der Schmelzpunkte und die Breiten der verbotenen Zonen. Wegen der Resonanzverfestigung liegen die Schmelzpunkte der Verbindungen höher als die der entsprechenden Elemente. Ebenso sind die Breiten der verbotenen Zonen größer als bei den entsprechenden Elementen, wobei jedoch festzustellen ist, daß die Vergrößerung der Breite der verbotenen Zone stärker ist als die Erhöhung des Schmelzpunktes. Während nämlich für die Erhöhung des Schmelzpunktes die Zunahme der Bindungsenergie schlechthin maßgeblich ist, ist es für die Zunahme der Breite der verbotenen Zone noch von besonderer Bedeutung, daß der Ionenanteil in der Bindung beim Übergang vom Element zurVerbindung $\mathrm{A}^{\mathrm{III}} \mathrm{B}^{\mathrm{V}}$ zunimmt. Bei sonst gleichen Verhältnissen (Bindungskräfte, Dichten usw.) hat nämlich ein reiner Ionenkristall eine viel größere Breite der verbotenen Zone als ein homöopolarer Kristall. Man denke z. B. an die durchsichtigen Alkalihalogenidkristalle mit verbotenen Zonen von der Größenordnung $10 \mathrm{eV}$ im Gegensatz zu den homöopolaren Elementen der vierten Gruppe mit verbotenen Zonen von der Größenordnung $1 \mathrm{eV}$. Der Grund für dieses unterschiedliche Verhalten liegt darin, daß im Ionenkristall die Elektronendichte zwischen den Atomen auf sehr kleine Werte absinkt. Dadurch wird der Potentialunterschied, d. h. die Differenz Maximum minus Minimum im periodischen Kristallpotential sehr groß. Beim homöopolaren Kristall sind diese Unterschiede viel kleiner, da die elektronischen Brücken zwischen den Gitterbausteinen glättend auf das periodische Potential wirken.

Da, wie bereits eingangs erwähnt, die Breite der verbotenen Zone proportional zu der Größe des maximalen Potentialunterschiedes im periodischen Kristallpotential ist, erklärt sich damit die unterschiedliche Breite der verbotenen Zone bei homöopolaren und hete̊ropolaren Kristallen.

Messungen haben nun ergeben, daß der Schmelzpunkt von InSb bei $796^{\circ} \mathrm{K}$ und die Breite der verbotenen Zone 0,53 eV ist, während bei grauem $\mathrm{Sn}$ der Schmelzpunkt mit etwa $500^{\circ} \mathrm{K}$ und die Breite der verbotenen Zone mit etwa $0,1 \mathrm{eV}$ angesetzt werden kann. Diese Zahlenwerte passen ausgezeichnet zu den oben erwähnten Erörterungen.

Die bisherigen Überlegungen können auch zu folgendem Ergebnis zusammengefaßt werden: Verglichen mit den Elementen der vierten Gruppe besitzen die Verbindungen $\mathrm{A}^{\mathrm{III}} \mathrm{B}^{\mathrm{V}}$, gemessen an ihren Schmelzpunkten, relativ große Breiten der verbotenen Zone. Dies ist ein wesentlicher Vorteil für die Herstellung dieser Körper.

Die bereits als Halbleiter bekannten Verbindungen vom Typ $\mathrm{A}^{\mathrm{II}} \mathrm{B}^{\mathrm{VI}}$ (z. B. $\mathrm{ZnS}$ ) würden wegen ihres noch ausgeprägteren Ionencharakters den eben genannten Vorteil in stärkerem Maße besitzen. Die Verbreiterung der verbotenen Zone ist hier jedoch schon so groß, daß sich diese Verbindungen im thermischen Gleichgewicht bereits den Isolatoren nähern. Deshalb finden diese Körper in erster Linie im Zusammenhang mit optischen Phänomenen eine Anwendung. Der hier schon stärkere Einfluß des Ionenanteils in der chemischen Bindung äußert sich auch in der Elektronenbeweglichkeit. Diese ist bei den Körpern $\mathrm{A}^{\mathrm{II}} \mathrm{B}^{\mathrm{VI}}$ lediglich von der Größenordnung $100 \mathrm{~cm}^{2} /$ Volt sec. Bei einem reinen Ionenkristall wie z. B. $\mathrm{NaCl}$ ist ja die Elektronenbeweglichkeit besonders klein (von der Größenordnung $10^{-3} \mathrm{~cm}^{2} /$ Volt sec). DieVerbindungen $\mathrm{A}^{\mathrm{III}} \mathrm{B}^{\mathrm{V}}$ nehmen also in elektrischer Hinsicht eine Zwisehenstellung zwischen den Elementen der vierten Gruppe und den Verbindungen $\mathrm{A}^{\mathrm{II}} \mathrm{B}^{\mathrm{VI}}$ ein, wobei die Elektronenbeweglichkeit Maximalwerte erreicht.

Bezüglich der Beeinflussung der Leitungseigenschaften durch Fremdstörstellen ist in Analogie zu den Elementen der vierten Gruppe zu erwarten, daß die Elemente der zweiten Gruppe wie Cd, $\mathrm{Zn}$ als Acceptoren, die der sechsten Gruppe wie Se, Te als Donatoren wirksam sind. In der Tat hat sich auch gezeigt, daß Se-Zusatz zu AlSb die sonst p-leitenden 
Proben n-leitend machte. Dabei konnte der Leitungstyp durch die Polarität dès Gleichrichtereffektes festgestelltwerden, da sich herausgestellt hat, daß dieVerbindungen $\mathrm{A}^{\mathrm{III}} \mathrm{B}^{\mathrm{V}}$ einen ausgeprägten Gleichrichtereffekt besitzen. Zwei typische Gleichrichterkurven von AlSb sind in Abb. 3 und 4 dargestellt.

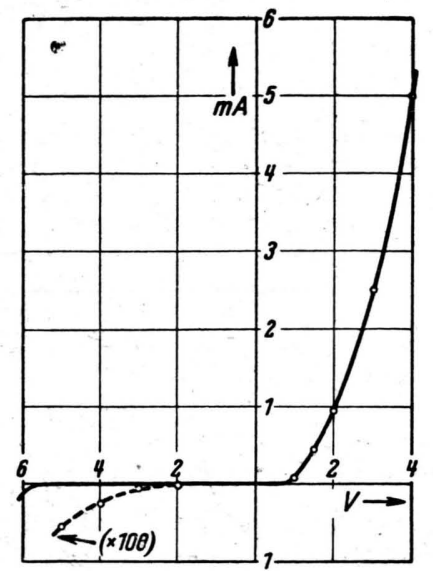

Abb. 3. Gleichrichterkennlinie eines n-leitenden AlSbKristalls mit Spitzenkontakt.

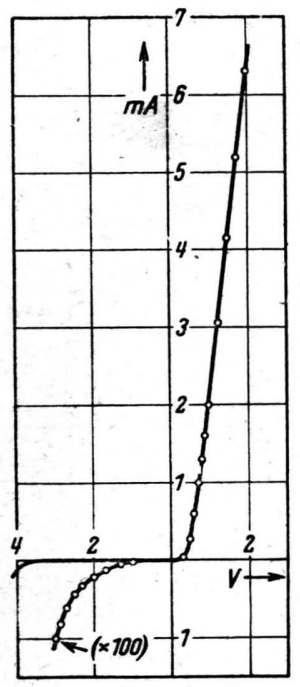

Abb. 4.Gleichrichterkennlinie eines p-leitend. AlSb-Kristalls mit Spitzenkontakt.
Besonders interessant ist es, die Wirkung von Fremdstörstellen aus der vierten Gruppe des periodischen Systems z. B. von $\mathrm{Pb} \mathrm{zu}$ studieren, welche vom Valenzstandpunkt aus betrachtet sowohl auf $\mathrm{A}^{3+}$-Plätzen als Donatoren als auch auf $\mathrm{B}^{5+}$-Plätzen als Acceptoren eingebaut werden können. Hier entscheidet dann das Ionenvolumen über den tatsächlich einzunehmenden Platz. So ist zu erwarten, daß das große $\mathrm{Pb}^{4+}$-Ion im InSb auf einen $\mathrm{In}^{3}+$.Platz eingebaut wird, hingegen im AlSb auf einen $\mathrm{Sb}^{5}+$.Platz. Die bisherigen Versuche sind mit dieser Vorstellung im Einklang.
Verbindungen unterscheiden sich von Elementen grundsätzlich dadurch, daß ein Atom A auf einem B-Platz sitzen kann und dadurch eine Art von Gitterstörung möglich wird, die selbst bei sorgfältigster Reinigung der Ausgangsstoffe nicht beseitigt werden kann. Dazu ist nun zu bemerken - und die Versuche haben dies bestätigt - , daß es viel schwieriger ist, eine $\mathrm{A}^{\mathrm{III}}$-Störstelle auf einem $\mathrm{B}^{\mathrm{V}}$-Platz einzubauen als etwa eine Störstelle aus der vierten oder sechsten Gruppe des periodischen Systems, da sie in jeder Hinsicht dem $\mathbf{B}^{\mathbf{V}}$-Atom viel weniger verwandt ist als die letztgenannten Elemente. Dies führt zu einer Unlöslichkeit der Komponenten A ${ }^{\text {III }}$ oder $\mathrm{B}^{\mathbf{V}}$ in der stöchiometrisch zusammengesetzten Verbindung $\mathrm{A}^{\mathrm{III}} \mathrm{B}^{\mathrm{V}}$ und ist gleichzeitig die Voraussetzung dafür, daß die stöchiometrisch zusammengesetzte Verbindung überhaupt hergestellt werden kann. In der Tat ist es bei der Verbindung InSb gelungen, die Verbindung so stöchiometrisch herzustellen, daß schon bei Zimmertemperatur Eigenleitung vorliegt.

Wie bereits erwähnt, besitzen die Verbindungen $\mathrm{A}^{\mathrm{III}} \mathrm{B}^{\mathrm{V}}$ einen ausgeprägten Gleichrichtereffekt. Insbesondere wurde sowohl der für n- als auch p-Leitung charakteristische Effekt festgestellt. Zusammen mit ihrer hohen Elektronenbeweglichkeit sind also die Verbindungen $\mathrm{A}^{\mathrm{III}} \mathrm{B}^{\mathrm{V}}$ geeignete Ausgangsstoffe für p-n-Gleichrichter und Transistoren.

Die praktische Herstellung der Verbindungen $\mathrm{A}^{\mathrm{III}} \mathrm{B}^{\mathrm{V}}$ erfolgt im allgemeinen durch Zusammenschmelzen der Komponenten. In ihren Kristallisationseigenschaften haben diese Verbindungen große Ähnlichkeit mit dem Germanium und dem Silicium.

Herrn Professor F. Trendelenburg fühle ich mich zu großem Dank verpflichtet für die energische Unterstützung, welche er dieser Arbeit von Anfang an angedeihen ließ.

An den experimentellen Untersuchungen waren meine Mitarbeiter, die Herren R. Grimm, O. Madelung und $H$. Weiß maßgeblich beteiligt, denen ich auch an dieser Stelle für ihre Hilfe bestens danken möchte. 